\title{
Risk Factors and Effect of Acute Kidney Injury on Outcomes Following Total Hip and Knee Arthroplasty
}

\author{
Zachary S. Aman \\ Thomas Jefferson University, zachary.aman@students.jefferson.edu \\ Michael Yayac \\ Thomas Jefferson University, michael.yayac@jefferson.edu \\ Alexander J. Rondon \\ Thomas Jefferson University, alexander.rondon@jefferson.edu \\ Timothy L. Tan \\ Thomas Jefferson University, timothy.tan@jefferson.edu \\ P. Maxwell Courtney \\ Thomas Jefferson University \\ Follow this and additional works at: https://jdc.jefferson.edu/si_ctr_2023_phase1 \\ Part of the Translational Medical Research Commons

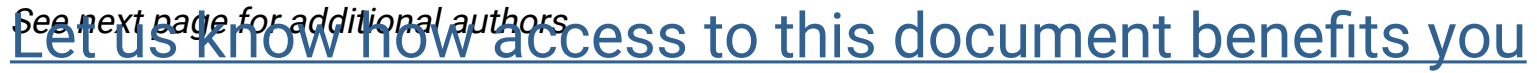

\section{Recommended Citation}

Aman, Zachary S.; Yayac, Michael; Rondon, Alexander J.; Tan, Timothy L.; Courtney, P. Maxwell; and Purtill, James J., "Risk Factors and Effect of Acute Kidney Injury on Outcomes Following Total Hip and Knee Arthroplasty" (2021). Phase 1. Paper 2.

https://jdc.jefferson.edu/si_ctr_2023_phase1/2

This Article is brought to you for free and open access by the Jefferson Digital Commons. The Jefferson Digital Commons is a service of Thomas Jefferson University's Center for Teaching and Learning (CTL). The Commons is a showcase for Jefferson books and journals, peer-reviewed scholarly publications, unique historical collections from the University archives, and teaching tools. The Jefferson Digital Commons allows researchers and interested readers anywhere in the world to learn about and keep up to date with Jefferson scholarship. This article has been accepted for inclusion in Phase 1 by an authorized administrator of the Jefferson Digital Commons. For more information, please contact: JeffersonDigitalCommons@jefferson.edu. 


\section{Authors}

Zachary S. Aman, Michael Yayac, Alexander J. Rondon, Timothy L. Tan, P. Maxwell Courtney, and James J. Purtill 
Sidney Kimmel

Medical College.

at Thomas Jefferson University

\section{Risk Factors and Effect of Acute Kidney Injury on Outcomes Following Total Hip and Knee Arthroplasty}

Zachary S. Aman, BA, Michael Yayac, MD, Alexander J. Rondon, MD, Timothy

L. Tan, MD, P. Maxwell Courtney, MD, James J. Purtill, MD*

THE JOURNAL OF ARTHROPLASTY

(9) AAHKS
> J Arthroplasty. 2020 Aug 1;S0883-5403(20)30865-2. doi: 10.1016/j.arth.2020.07.072. Online ahead of print.

Risk Factors and Effect of Acute Kidney Injury on Outcomes Following Total Hip and Knee Arthroplasty

Michael Yayac ${ }^{1}$, Zachary S Aman ${ }^{2}$, Alexander J Rondon ${ }^{3}$, Timothy L Tan ${ }^{3}$, P Maxwell Courtney

1 , James J Purtill ${ }^{1}$

Affiliations + expand

PMID: 32839060 DOI: 10.1016/j.arth.2020.07.072 


\section{Sidney Kimmel}

Medical College.

at Thomas Jefferson University

\section{Introduction}

- Acute Kidney Injury

- Abrupt decrease in kidney function due to structural or functional damage':

- Several etiologies: ischemia, nephrotoxicity, obstruction, etc.

- Known to significantly complicate clinical course and worsen outcomes in hospitalized patients

- AKI After TJA

- 2- $15 \%$ of THA and TKA patients ${ }^{2}$

- Potentially avoidable complication related to negative outcomes

- longer LOS, increased readmission rates, and increased healthcare costs ${ }^{3}$

- Can lead to CKD2

- Risk Factors for AKI

- Increased age, CHF, postoperative hypotension, and hepatic failure ${ }^{4-6}$

- In post-cardiac surgery patients, anemia has been reported to be a risk factor for $\mathrm{AKI}^{7-10} \rightarrow$ medullary hypoxia ${ }^{11}$

- Gharaibeh et al reported that for every $5-\mathrm{kg} / \mathrm{m} 2$ increase in BMI, patients had a $40 \%$ increase risk of AKI post-THA ${ }^{12}$ 


\section{Introduction}

- There remains limited information regarding the association of modifiable risk factors and AKI post-TJA

- Anemia, length of surgery, blood loss, antibiotics, etc.

- How can surgeons better identify and proactively mitigate modifiable risk factors of AKI?

- Risk reduction can lead to improved outcomes and lower costs 


\section{Purpose $\&$ Hypothesis}

- Purpose:

-1)To determine the effect of AKI on short-term outcomes

- 2) to identify risk factors for developing AKI following THA and TKA

- Hypothesis:

-1) AKI has significant adverse effects on shortterm outcomes metrics including length of stay, discharge, and complications

- 2) Increased BMI and inadequate intraoperative renal perfusion are modifiable risk factors associated with postoperative AKI 


\section{Methods: Overview}

- Study design: Retrospective Case Control

- Data source and collection: Rothman Orthopedic Institute Database

- Population/study sample: Patients undergoing THA or TKA between 2005 and 2017 with minimum 2-year $\mathrm{f} / \mathrm{u}$

- Intervention: THA or TKA

- Comparison group: Patients who developed AKI vs. Patients who did not post-op

- Outcome: 1) Risk factors for the development of AKI and 2) AKI effect

Patients undergoing THA or

TKA between 2005-2017

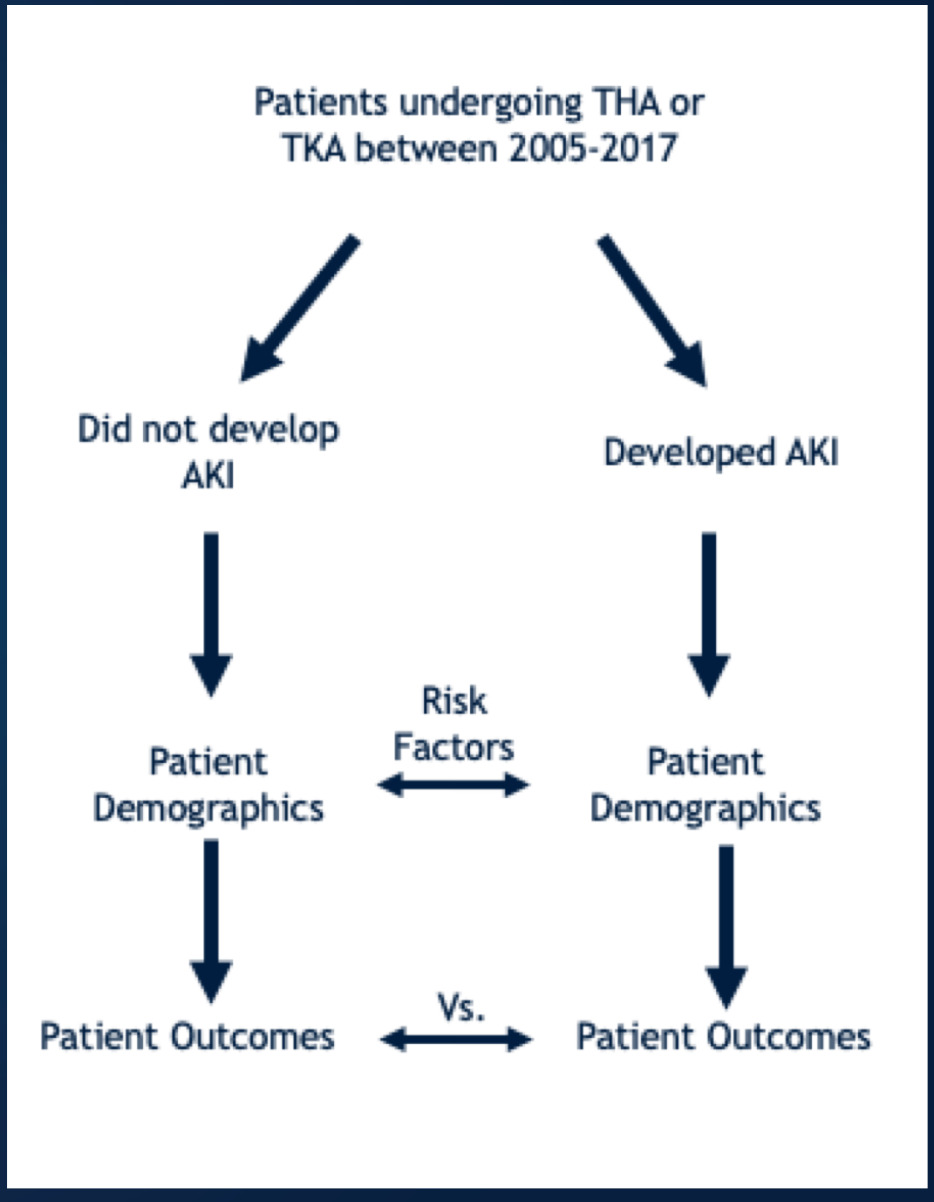
on post-operative pt. outcomes vs. controls 
- Data Collection

- Demographics

- Operative Variables

- Surgical time, antibiotic prophylaxis, EBL, Hgb

- Medical comorbidities

- CHF, dementia, CKD, DM, etc.

- Outcomes Measures

- Complications, LOS, discharge to facility, readmission, 90-day mortality

- Creatinine level measurement (pre/post)

- AKI defined as increased in serum creatinine by $50 \%$ or $0.3 \mathrm{mg} / \mathrm{dl}$ on postop day 1 from preop measurements 


\section{Methods: Analysis}

\section{Statistical analyses}

- performed to identify significant risk factors in patients who developed AKI and its effect on postoperative outcomes as compared to control

- Continuous variables $\rightarrow$ Mann-Whitney test

- Categorical variables $\rightarrow$ Chi-square analysis

- Independent effect of AKI on secondary outcomes $\rightarrow$ Multivariate regression 


\section{Sidney Kimmel}

Medical College.

at Thomas Jefferson University

\section{Results: Risk Factors}

Demographics

- 20,800 patients who underwent unilateral primary THA $(n=10,601)$ or TKA $(\mathrm{n}=10,199)$ from 2005 to 2017

- 814 (3.9\%) patients developed AKI vs. 19, 986 patients did not develop AKI

\section{Patient Risk Factors}

- Patients who developed AKI had significantly higher:

- Age (66.4 vs 63.4, $\mathrm{p}<0.001)$

- BMI (31.8 vs 30.0, $p<0.001)$

- Rates of 10 of the 17 assessed comorbidities (Appendix 1)

\section{Operative Risk Factors}

- Patients who developed AKI had:

- Significantly lower Preoperative Hgb levels (13.1 vs. 13.5, $\mathrm{p}<0.001$ ).

- more likely to undergo TKA (55\% vs $49 \%, p=0.002$ )

- Significantly longer operative times (90 vs. 79 min, $p<0.001)$,

- less likely to receive TXA ( $14 \%$ vs. $22 \%, p<0.001)$,

- Received greater intraoperative $R B C$ transfusion units $(2.1$ vs $0.4, p=0.015)$ 


\section{Sidney Kimmel}

Medical College.

at Thomas Jefferson University

\section{Results: Outcomes}

\section{Clinical Outcomes}

- Patients developing AKI had significantly higher:

- rates of all in-hospital complication types except for gastrointestinal complications

- greater LOS (3.6 vs 2.8 days, $\mathrm{p}<0.001$ )

- discharge to facility rates ( $37 \%$ vs $23 \%, p<0.001)$

- 90-day mortality rates ( $1 \%$ vs. $0.1 \%, p<0.001)$

- 1-year $\mathrm{PJI}$ rates $(1.8 \%$ vs. $0.7 \%, p=0.001)$

Multivariate Analysis

- AKI significantly increased

- LOS by 0.26 days $(95 \% \mathrm{Cl} 0.14-0.38, \mathrm{p}<0.001)$.

- Risk of in-hospital complications (OR=1.73, 95\% Cl 1.452.07, $\mathrm{p}<0.001$ )

- Risk of being discharged to a facility (OR=1.26, 95\% Cl 1.05$1.53, p=0.012$ ) 


\section{Conclusions}

- AKI has significant adverse effects on shortterm outcomes metrics including length of stay, discharge to a facility, and increased rate of in-hospital complications

- While most risk factors for developing AKI may not be modifiable, efforts to reduce BMI preoperatively and maintaining adequate renal perfusion (anemia, estimated blood loss, and length of surgery) intraoperatively may aid in mitigating this risk. 


\section{Future Directions}

- Future studies should be performed to determine if employing strategies to address modifiable risk factors pre- and intraoperatively reduce the incidence of AKI in patients undergoing TJA

- Treatment of anemia prior to TJA may be beneficial to prevent AKI

- Efforts to limit blood loss, such as using TXA, which demonstrated a protective effect in our analysis, may be effective in reducing risk of AKI 


\section{References}

- [1] Kidney Disease: Improving Global Outcomes (KDIGO) Acute Kidney Injury Work Group. KDIGO Clinical Practice Guideline for Acute Kidney Injury. Kidney inter., Suppl. 2012; 2: 1-138. n.d.

- [2] Rudy MD, Ahuja NK. Acute Kidney Injury in Lower-Extremity Joint Arthroplasty: Clinical Epidemiology, Prevention, and Management. JBJS Reviews 2019;7:e9. https://doi.org/10.2106/JBJS.RVW.18.00121.

- [3] Abar O, Toossi N, Johanson N. Cost and determinants of acute kidney injury after elective primary total joint arthroplasty. Arthroplast Today 2018;4:335-9. https://doi.org/10.1016/j.artd.2018.05.002.

- [4] Lands VW, Malige A, Carmona A, Roscher CR, Gayner RS, Rowbotham J, et al. Reducing Hypotension and Acute Kidney Injury in the Elective Total Joint Arthroplasty Population: A Multi-Disciplinary Approach. The Journal of Arthroplasty 2018;33:1686-92. https://doi.org/10.1016/j.arth.2018.01.061.

- [5] Weingarten TN, Gurrieri C, Jarett PD, Brown DR, Berntson NJ, Calaro RD, et al. Acute kidney injury following total joint arthroplasty: retrospective analysis. Can J Anaesth 2012;59:1111-8. https://doi.org/10.1007/s12630-012-9797-2.

- [6] Warth LC, Noiseux NO, Hogue MH, Klaassen AL, Liu SS, Callaghan JJ. Risk of Acute Kidney Injury After Primary and Revision Total Hip Arthroplasty and Total Knee Arthroplasty Using a Multimodal Approach to Perioperative Pain Control Including Ketorolac and Celecoxib. J Arthroplasty 2016;31:253-5. https://doi.org/10.1016/j.arth.2015.08.012.

- [7] Karkouti K, Wijeysundera DN, Yau TM, McCluskey SA, Chan CT, Wong P-Y, et al. Influence of erythrocyte transfusion on the risk of acute kidney injury after cardiac surgery differs in anemic and nonanemic patients. Anesthesiology 2011;115:523-30. https://doi.org/10.1097/ALN.0b013e318229a7e8.

- [8] Karkouti K, Grocott HP, Hall R, Jessen ME, Kruger C, Lerner AB, et al. Interrelationship of preoperative anemia, intraoperative anemia, and red blood cell transfusion as potentially modifiable risk factors for acute kidney injury in cardiac surgery: a historical multicentre cohort study. Can J Anaesth 2015;62:377-84. https://doi.org/10.1007/s12630014-0302-y.

- [9] Walsh M, Garg AX, Devereaux PJ, Argalious M, Honar H, Sessler DI. The association between perioperative hemoglobin and acute kidney injury in patients having noncardiac surgery. Anesth Analg 2013;117:924-31. https://doi.org/10.1213/ANE.0b013e3182a1ec84.

- [10] Rosenberger C, Rosen S, Heyman SN. Renal parenchymal oxygenation and hypoxia adaptation in acute kidney injury. Clin Exp Pharmacol Physiol 2006;33:980-8. https://doi.org/10.1111/j.1440-1681.2006.04472.x.

- [11] Park JT. Postoperative acute kidney injury. Korean J Anesthesiol 2017;70:258-66. https://doi.org/10.4097/kjae.2017.70.3.258.

- $\quad$ [12] Gharaibeh KA, Hamadah AM, Sierra RJ, Leung N, Kremers WK, El-Zoghby ZM. The Rate of Acute Kidney Injury After Total Hip Arthroplasty Is Low but Increases Significantly in Patients with Specific Comorbidities: The Journal of Bone and Joint Surgery 2017;99:1819-26. 
Sidney Kimmel Medical College. at Thomas Jefferson University

Appendix 1. Patient Demographics and

Comorbidities by groups of 'AKI'

\section{Appendix.}

\begin{tabular}{|c|c|c|c|}
\hline & $\begin{array}{c}\text { No } \\
N=19986\end{array}$ & $\begin{array}{c}\text { Yes } \\
N=814\end{array}$ & p-value \\
\hline Patient Age & $63.4(11.3)$ & $66.4(11.1)$ & $<0.001$ \\
\hline Body Mass Index & $30.0(5.73)$ & $31.8(6.20)$ & $<0.001$ \\
\hline Sex & & & $<0.001$ \\
\hline Female & $11171(55.9 \%)$ & $367(45.1 \%)$ & \\
\hline Male & $8815(44.1 \%)$ & $447(54.9 \%)$ & \\
\hline \multicolumn{4}{|l|}{ Medical Comorbidities } \\
\hline Anemia & $6777(34.1 \%)$ & $433(53.4 \%)$ & $<0.001$ \\
\hline AIDS & $17(0.09 \%)$ & $3(0.37 \%)$ & 0.041 \\
\hline Congestive Heart Failure & $336(1.68 \%)$ & $50(6.14 \%)$ & $<0.001$ \\
\hline Chronic Pulmonary Disease & $2392(12.0 \%)$ & $125(15.4 \%)$ & 0.004 \\
\hline Cerebrovascular Disease & $230(1.15 \%)$ & $20(2.46 \%)$ & 0.001 \\
\hline Dementia & $27(0.14 \%)$ & $5(0.61 \%)$ & 0.008 \\
\hline Diabetes Mellitus & $252(1.26 \%)$ & $22(2.70 \%)$ & 0.001 \\
\hline Hemiparesis & $6(0.03 \%)$ & $1(0.12 \%)$ & 0.244 \\
\hline Malignancy & $218(1.09 \%)$ & $14(1.72 \%)$ & 0.132 \\
\hline Metastatic Disease & $45(0.23 \%)$ & $3(0.37 \%)$ & 0.437 \\
\hline Myocardial Infarction & $844(4.22 \%)$ & $67(8.23 \%)$ & $<0.001$ \\
\hline Mild Liver Disease & $90(0.45 \%)$ & $6(0.74 \%)$ & 0.280 \\
\hline Moderate-Severe Liver Disease & $12(0.06 \%)$ & $2(0.25 \%)$ & 0.102 \\
\hline Peptic Ulcer Disease & $73(0.37 \%)$ & $5(0.61 \%)$ & 0.234 \\
\hline Peripheral Vascular Disease & $261(1.31 \%)$ & $24(2.95 \%)$ & $<0.001$ \\
\hline Chronic Kidney Disease & $315(1.58 \%)$ & $74(9.09 \%)$ & $<0.001$ \\
\hline Connective Tissue Disease & $650(3.25 \%)$ & $32(3.93 \%)$ & 0.334 \\
\hline Charlson Comorbidity Index & $0.40(0.88)$ & $0.84(1.43)$ & $<0.001$ \\
\hline
\end{tabular}


Sidney Kimmel Medical College. at Thomas Jefferson University

\section{Appendix.}

Appendix 1 (continued). Patient Demographics and

Comorbidities by groups of 'AKI'

\begin{tabular}{|c|c|c|c|}
\hline & $\begin{array}{c}\text { No } \\
N=19986\end{array}$ & $\begin{array}{c}\text { Yes } \\
N=814\end{array}$ & p-value \\
\hline Tobacco Use & & & $<0.001$ \\
\hline Current & $1830(9.16 \%)$ & $52(6.39 \%)$ & 0.007 \\
\hline Former & $6821(34.1 \%)$ & $333(40.9 \%)$ & $<0.001$ \\
\hline None & $11334(56.7 \%)$ & $429(52.7 \%)$ & 0.024 \\
\hline Illicit Drug Use & & & 0.203 \\
\hline No & $19557(97.9 \%)$ & $798(98.0 \%)$ & \\
\hline Former & $250(1.25 \%)$ & $13(1.60 \%)$ & \\
\hline Yes & $178(0.89 \%)$ & $3(0.37 \%)$ & \\
\hline Preoperative Creatinine (mg/dL) & $0.90(0.30)$ & $1.11(1.11)$ & $<0.001$ \\
\hline Preoperative BUN (mg/dL) & $17.7(6.30)$ & $20.2(8.05)$ & $<0.001$ \\
\hline Preoperative Hemoglobin (g/dL) & $13.5(1.41)$ & $13.1(1.62)$ & $<0.001$ \\
\hline
\end{tabular}


Sidney Kimmel Medical College. at Thomas Jefferson University

\section{Appendix.}

Appendix 2. Outcomes by groups of 'AKI'

\begin{tabular}{lccc}
\hline & No & Yes & p-value \\
\hline Postoperative Creatinine & $0.87(0.27)$ & $1.59(1.28)$ & 0.000 \\
Postoperative BUN & $13.2(5.62)$ & $23.3(9.32)$ & $<0.001$ \\
Postoperative Hemoglobin & $10.4(1.43)$ & $9.82(1.49)$ & $<0.001$ \\
Complication & $3416(17.1 \%)$ & $267(33.0 \%)$ & $<0.001$ \\
Cardiovascular Complications & $656(3.28 \%)$ & $45(5.53 \%)$ & 0.001 \\
DVT/PE & $152(0.76 \%)$ & $15(1.84 \%)$ & 0.001 \\
Pulmonary Complications & $148(0.74 \%)$ & $18(2.21 \%)$ & $<0.001$ \\
GI Complications & $1153(5.77 \%)$ & $41(5.04 \%)$ & 0.422 \\
GU Complications & $300(1.50 \%)$ & $90(11.1 \%)$ & $<0.001$ \\
Neurological Complications & $182(0.91 \%)$ & $17(2.09 \%)$ & 0.001 \\
Infectious Complications & $210(1.05 \%)$ & $17(2.09 \%)$ & 0.009 \\
Discharge to Facility & $4666(23.4 \%)$ & $301(37.4 \%)$ & $<0.001$ \\
Length of Stay & $2.76(2.37)$ & $3.59(2.59)$ & $<0.001$ \\
Readmission & $793(3.97 \%)$ & $42(5.16 \%)$ & 0.108 \\
90-day Mortality & $28(0.14 \%)$ & $8(0.98 \%)$ & $<0.001$ \\
1-year PJI & $143(0.72 \%)$ & $15(1.84 \%)$ & $\mathbf{0 . 0 0 1}$ \\
\hline
\end{tabular}



Medical College at Thomas Jefferson University

\section{Appendix.}

Appendix 3. Relative Variable Importance

Body Mass IndexPostoperative HemoglobinPreoperative CreatininePreoperative Hemoglobin-
Preoperative BUNPreoperative BUNCrystalloid Fluids Given-

$$
\text { Alcohol Use- }
$$

Charlson Comorbidity IndexPatient Age-

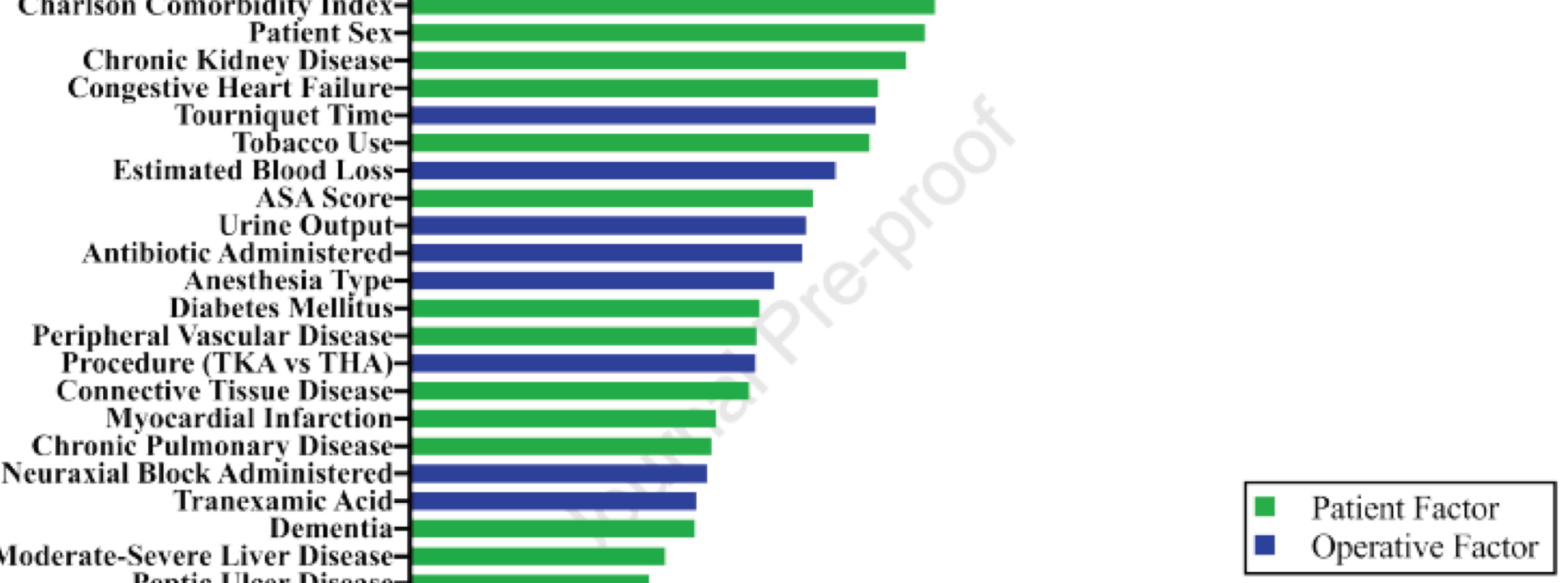

Moderate-Severe Liver Disease-

Peptic Ulcer DiseaseCancer Illicit Drug Use-
Cerebrovascular DiseaseIntraoperative Blood SalvageMild Liver Disease-

Metastatic Disease Allogeneic Blood TransfusionHemiparesisColloid Fluids Give

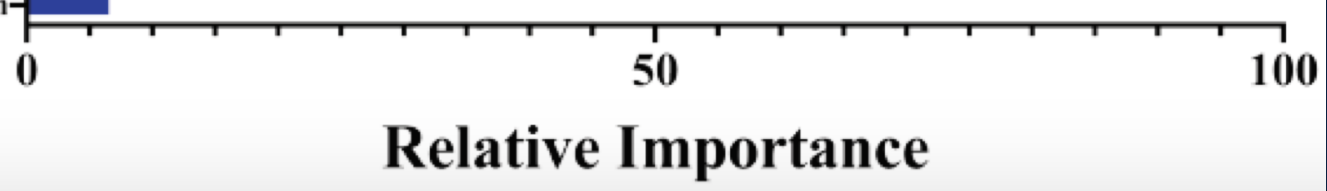

